\section{End-of-treatment and serial PET imaging has \\ prognostic value and clinical utility in primary mediastinal B-cell lymphoma following dose-adjusted EPOCH-R - Response to Adams et al.}

We recently published additional and extended followup results from our prospective phase 2 study and independent series of DA-EPOCH-R in the front-line treatment of primary mediastinal B-cell lymphoma (PMBCL). ${ }^{1}$ In this study, analyses of singular and serial end-of-treatment (EOT) FDG-PET imaging was included in an attempt to better define the predictive ability of such imaging in PMBCL patients following DA-EPOCH-R. In response to our paper, Adams and Kwee noted several limitations to our study and call into question the value of EOT and serial FDG-PET imaging given the overall favorable prognosis and low incidence of relapse in PMBCL and the unproven survival benefit with such imaging. Although we recognize these limitations, we disagree with their conclusions and believe that EOT serial FDG-PET imaging does indeed have clinical utility in PMBCL patients following DA-EPOCH-R.

One of the main benefits of dose-intensive therapy, such as DA-EPOCH-R, is its ability to obviate the need for radiotherapy in most patients with $\mathrm{PMBCL} .{ }^{2} \mathrm{~A}$ recent retrospective, multicenter study claimed comparability of $\mathrm{R}-\mathrm{CHOP}$ to DA-EPOCH-R in the up-front treatment of PMBCL based on a similar 2-year rate of progression-free (PFS) and overall survival (OS), however, this "comparability" was at the expense of significantly greater radiotherapy use in R-CHOP treated patients (59\% vs. $13 \%$, $P<0.001) .^{3}$ Given the young median age in PMBCL and the significant long-term toxicity associated with routine radiotherapy use, accurate discrimination of patients who are cured following immuno-chemotherapy alone is critically important in order to spare these patients from the harmful effects of additional radiotherapy. EOT FDG-PET is one such technique that is frequently used in clinical practice for this purpose.

Of the 80 PMBCL patients with evaluable EOT FDGPET scans in our study, $55(69 \%)$ patients had negative (i.e., Deauville 1-3) scans with only 1 patient experiencing treatment failure (negative predictive value (NPV) of $98.2 \%) .{ }^{1}$ The high NPV of EOT FDG-PET as well as the favorable outcome in those with negative EOT scans is in line with other retrospective studies in PMBCL following DA-EPOCH- ${ }^{4}$ as well as the prospective IELSG-26 study which utilized combined-modality therapy. ${ }^{5}$ The low rate of treatment failure seen in our study is not appreciably different from other retrospective studies using DAEPOCH-R alone in PMBCL, ${ }^{4}$ and cannot be directly compared to studies utilizing alternative up-front treatment regimens such as R-CHOP. Despite the small number of treatment failures in our study, EOT FDG-PET did indeed have prognostic value with a lower rate of treatment failure $(2 \%$ vs. $20 \%)$ and a significant improvement in PFS and $O S$ in those PMBCL patients with a negative EOT scan following DA-EPOCH-R.

More important are the results obtained from the patients in our study with positive EOT FDG-PET scans. Of the 25 patients with positive (i.e., Deauville 4-5) EOT FDG-PET scans, 20 (80\%) remained progression-free after long-term follow up, highlighting the poor positive predictive value (PPV) and high false-positive rate seen with EOT FDG-PET imaging in this patient population. ${ }^{1}$ Common clinical practice in PMBCL, however, is to consider a positive EOT FDG-PET scan indicative of active residual disease and need of further radiotherapy.
Indeed, even following DA-EPOCH-R, retrospective studies have shown that PMBCL patients with a positive EOT FDG-PET scan are more likely to receive additional radiotherapy than those with negative scans $(38.9 \%$ vs. $6.5 \%, P<0.001) .{ }^{4}$ Given the high false-positive rate of EOT FDG-PET scans and difficulty in obtaining confirmatory biopsy in all cases, routine radiotherapy use in all patients with positive EOT scans following DA-EPOCH$\mathrm{R}$ will result in significant overtreatment of patients, subjecting them to an increased risk of premature death from second malignancies and cardiovascular disease from radiotherapy.

In regard to the serial FDG-PET analysis, we do recognize that the small number of treatment failures limit the conclusions that can be drawn. We also acknowledge that it is impossible to conclude that the 2 positive EOT FDG-PET treatment failures without confirmatory biopsy had true active disease. Both of these patients, however, met radiographic criteria for disease progression with interval growth on CT and a progressive increase in SUVmax with new FDG-avid lesions on FDG-PET, respectively, both justification for salvage radiotherapy implementation. Nonetheless, the main focus of our serial FDG-PET analysis was not to identify early the small number of treatment failures but was more to identify the larger number of false-positive non-progressors. Despite a Deauville score of 4-5 and a median (range) SUV $_{\max }$ of $4.7(2.8-11.4)$ in the positive EOT FDG-PET non-progressors, linear regression of serial FDG-PET scans in these patients demonstrated an overall decrease in $\mathrm{SUV}_{\text {max }}$ compared to an increase in treatment failures. We agree that routine surveillance imaging in unselected patients with DLBCL has not clearly led to improved survival, 8,9 but the intent of serial FDG-PET imaging in our study was not to show improved survival with salvage in treatment failures but was to reduce unnecessary radiotherapy in non-progressors. Indeed, serial FDG-PET imaging did allow us to discriminate those false-positive patients without treatment failure and reduce radiotherapy use by $80 \%$ from 25 patients with positive EOT scans to only 5 patients with treatment failure.

In conclusion, EOT FDG-PET has clear prognostic value in PMBCL patients following front-line treatment with DA-EPOCH-R. PMBCL patients who achieve a negative EOT FDG-PET following DA-EPOCH-R have a low risk of subsequent treatment failure and should not undergo additional FDG-PET imaging nor should receive radiotherapy. A singular positive EOT FDG-PET following DA-EPOCH-R is not indicative of residual disease in most PMBCL patients and more likely represents posttreatment inflammatory changes. Serial FDG-PET imaging has clinical utility in identification of false-positive non-progressors who can be spared the long-term toxicity associated with radiotherapy and thus should be considered in all PMBCL patients with an initial positive EOT scan following DA-EPOCH-R.

Christopher Melani, Mark Roschewski and Wyndham H. Wilson

Lymphoid Malignancies Branch, Center for Cancer Research, National Cancer Institute, National Institutes of Health, Bethesda, MD, USA

Correspondence:wilsonw@mail.nih.gov or wyndham.wilson@gmail.com doi:10.3324/haematol.2018.199547

Information on authorship, contributions, and financial \& other disclosures was provided by the authors and is available with the online version of this article at www. haematologica.org. 


\section{References}

1. Melani C, Advani R, Roschewski M, et al. End-of-treatment and serial PET imaging in primary mediastinal B-cell lymphoma following dose-adjusted-EPOCH-R: A paradigm shift in clinical decision making. Haematologica. 2018 May 10. [Epub ahead of print]

2. Dunleavy K, Pittaluga S, Maeda LS, et al. Dose-adjusted EPOCH-rituximab therapy in primary mediastinal B-cell lymphoma. N Engl J Med. 2013;368(15):1408-1416.

3. Shah NN, Szabo A, Huntington SF, et al. R-CHOP versus doseadjusted R-EPOCH in frontline management of primary mediastinal B-cell lymphoma: a multi-centre analysis. $\mathrm{Br} \mathrm{J}$ Haematol. 2018;180(4):534-544.

4. Giulino-Roth L, O'Donohue T, Chen Z, et al. Outcomes of adults and children with primary mediastinal B-cell lymphoma treated with dose-adjusted EPOCH-R. Br J Haematol. 2017;179(5):739-747.

5. Martelli M, Ceriani L, Zucca E, et al. [18F]fluorodeoxyglucose positron emission tomography predicts survival after chemoim- munotherapy for primary mediastinal large B-cell lymphoma: results of the International Extranodal Lymphoma Study Group IELSG-26 Study. J Clin Oncol. 2014;32(17):1769-1775.

6. Schaapveld M, Aleman BM, van Eggermond AM, et al. Second cancer risk up to 40 years after treatment for Hodgkin's lymphoma. $\mathrm{N}$ Engl J Med. 2015;373(26):2499-2511.

7. Bhakta N, Liu Q, Yeo F, et al. Cumulative burden of cardiovascular morbidity in paediatric, adolescent, and young adult survivors of Hodgkin's lymphoma: an analysis from the St Jude Lifetime Cohort Study. Lancet Oncol. 2016;17(9):1325-1334.

8. Thompson CA, Ghesquieres H, Maurer MJ, et al. Utility of routine post-therapy surveillance imaging in diffuse large B-cell lymphoma. J Clin Oncol. 2014;32(31):3506-3512.

9. El-Galaly TC, Jakobsen LH, Hutchings M, et al. Routine imaging for diffuse large B-cell lymphoma in first complete remission does not improve post-treatment survival: a Danish-Swedish populationbased study. J Clin Oncol. 2015;33(34):3993-3998. 